\title{
SPECTROPOLARIMETRY AND WIND ASYMMETRIES OF
}

\section{WOLF-RAYET STARS}

\author{
REGINA E. SCHULTE-LADBECK \\ Department of Physics and Astronomy, University of Pittsburgh, \\ 3941 O'Hara Street, Pittsburgh, PA 15260, U.S.A.
}

\begin{abstract}
Motivated by the question of what drives the winds of Wolf-Rayet (WR) stars, I have been investigating the structure of WR winds. Observational evidence has been accumulated which demonstrates that the winds of some (but not all) WR stars are not spherically symmetric or homogeneous. I review results of work in the literature pertaining to the detection of such wind asymmetries in single WR stars and WR binary components. Particular emphasis is placed on the technique of spectropolarimetry, and how it has contributed to our knowledge of the wind-geometry of WR stars.
\end{abstract}

Key words: stars: Wolf-Rayet - polarization

\section{Motivation}

In radiation-driven wind theory successfully applied to O-type stars, radiative momentum arising from stellar luminosity is transferred to the stellar outflow through line scattering (e.g., Lucy \& Solomon 1970). During the last 15 years, it has been increasingly doubted that it might be possible to drive the winds of WR stars with radiation pressure as well, because their mechanical wind momentum often exceeds the single-scattering photon momentum by over an order of magnitude. This discrepancy has entered the literature as the "momentum problem" of WR winds (but see Owocki, these proceedings, who shows that it is actually an "opacity problem").

Three avenues of research have subsequently been pursued to solve the "momentum problem": Underhill \& Fahey (1987a) questioned radiative driving, and instead they have assumed that non-radiative energy and momentum owing to strong pre-natal magnetic fields are deposited in WR winds. They suggested that the "momentum problem" would not exist if the massloss rates of WR stars were significantly lower than those derived by others. However, this idea has not found independent support.

A solution to the "momentum problem" within a purely radiation-driven wind theory requires the extraction of more momentum from the radiation field. This could, in principle, be achieved via multiple scattering. Lucy \& Abbott (1993) recently showed that a higher luminosity conversion due to multiple scattering exists in winds with strong ionization stratification (however, the ionization stratification which they assumed in their model has been criticized by Schmutz, these proceedings). Schulte-Ladbeck, Eenens \& Davis (in preparation) are currently investigating the ionization structure of two prototypical WR stars observationally. 
Alternatively, in the so-called hybrid models, an additional force is required to drive the winds of WR stars. One such hybrid model is the Luminous Magnetic Rotator (LMR) model by Poe, Friend \& Cassinelli (1989), in which a wind is driven out of the stellar equator by a combination of radiative, magnetic, and rotational forces.

The LMR model was being constructed when I was a postdoc at the University of Wisconsin. We quickly realized that, in the absence of photospheric absorption lines in most WR stars, there was little hope of providing direct observational proof for rapid rotation. The emission-line profiles can be interpreted both with and without the inclusion of rotation (see below). In addition, the slope of the optical and radio continuum does not reflect deviations from spherical geometry either (see Schmid-Burgk 1982; Doherty 1991). We concluded that the only readily observable consequence of the LMR model is polarization. Thus was started, about five years ago, an observational program to investigate the winds of WR stars with spectropolarimetry.

This paper is organized as follows. I first discuss theoretical implications of rotation on stellar wind parameters. Then, I review evidence for and against spherical winds that has been obtained by techniques other than spectropolarimetry. After that, I present the major wind geometries identified by spectropolarimetry. In the summary, I outline some implications of the data for the evolution of angular momentum in massive stars.

\section{Effects of asymmetries on derived wind properties}

Rotation has several effects on the structure of WR winds. It is expected to severely affect the wind characteristics, such as the velocity law and the mass-loss rate, thus potentially producing errors in the stellar parameters which are derived using spherical wind models.

A velocity law for a Castor, Abbott \& Klein (1975) wind with and without rotation was presented as early as 1979 by Castor. Apart from remarking that "the effect of stellar rotation on a radiatively driven stellar wind is a particularly unpleasant problem", he concluded that "the rise of velocity with radius is quite appreciably slower when rotation is included, and the terminal velocity is less". Owocki, Cranmer \& Blondin (1994, based on Bjorkman \& Cassinelli 1993, cf. Cassinelli, these proceedings) recently provided a modified Wind Compressed Disk model, in which the velocity law of a B2.5V star rotating at $350 \mathrm{~km} / \mathrm{s}$ is computed as a function of the inclination angle. There are significant differences in the shape of the velocity curve between the polar wind, which reaches a terminal speed of $1220 \mathrm{~km} / \mathrm{s}$, and the equatorial wind, which acquires a speed of $435 \mathrm{~km} / \mathrm{s}$.

Schmid-Burgk (1982) investigated the effects of asymmetries on stellar mass-loss rates. He showed that "deviations from spherical symmetry always 
lead to overestimates of the true mass-loss rate if one mistakes the wind to be spherically symmetric". Specifically, the median of a sample of randomly inclined objects is at an inclination of $60^{\circ}$, and overestimates by a factor of 2 are possible for structures with scale-length ratios of a factor of 10 , i.e., disks or cigars. High mass-loss rates can also be mimicked when the stellar wind consists of numerous, very narrow streamers, even when these are, on a large-scale average, distributed spherically symmetrically over the surface of the source. There is now evidence from polarimetry both for flattened wind distributions and for clumping within a time-averaged spherical WR wind ( $c f$. Schulte-Ladbeck, Meade \& Hillier 1992, and references therein).

\section{Arguments favoring spherical winds}

The spectra of WR stars display emission lines which harbor kinematic information on their winds. The broad lines are generally interpreted with Doppler broadened emission in the outflow. A major argument for the presence of spherical winds has been put forth by Kuhi (1973), who showed that the flat-topped profiles observed in many WR stars originate from spherically symmetric, hollow, expanding shells.

A search for orientation effects in WR binaries was conducted by Aslanov \& Cherepashchuk (1990). They used eclipsing binaries and binaries with known inclinations from polarization studies together with the assumption that the stellar spin axes are aligned with the orbital spin axes to test the hypothesis that numerous WR stellar parameters are latitude dependent. The negative results of this study led the authors to conclude that "the characteristics of the optical line emission and X-ray Bremsstrahlung from WR+OB binaries are not functions of their orbital inclination".

Robert (1991) has investigated the behavior of the sub-peaks of emission lines, identified as blobs or clumps in inhomogeneous winds. The trajectories of the ejected parcels do appear to indicate that the time-averaged geometries of several WR winds are spherical.

\section{Arguments favoring non-spherical winds}

WR stars are the evolutionary descendants of massive 0-type stars. There is direct observational evidence for high rotational speeds in $\mathrm{O}$ stars, from the width of their photospheric absorption lines. Conti \& Ebbets (1977) showed that $v \sin i$ ranges from a few ten $\mathrm{km} / \mathrm{s}$ to about $400 \mathrm{~km} / \mathrm{s}$ and conclude that "early type stars begin their (...) lives on the main sequence with a wide range of angular momentum". What happens to the angular momentum as $\mathrm{O}$-type stars evolve into WR stars? Little is known at present about exactly how angular momentum is conserved in massive stars, how it is transported through the interior of massive stars, and how it is lost 
in the stellar winds. The problem of determining the rotational speeds of WR stars lies in their very spectra. There are generally no absorption lines present that are considered to be photospheric (apart from a few WN7+abs cases, Crowther et al. 1994, for which there are no $v \sin i$ determinations).

One and only one WR star, WR138, has had its rotational speed measured from absorption lines profiles (Massey 1980; Schmutz 1990). Schmutz provided arguments favoring a single-star interpretation of the spectrum, derived a rotational speed of $450 \mathrm{~km} / \mathrm{s}$, and concluded that the WR star rotates close to break-up speed. However, this result has been questioned by Moffat at this meeting ( $c f$. the discussion following this paper), because WR138 has been shown to be the member of a long-period, $P=1538$ days, binary system ( $c f$. Annuk 1991 and references therein). Moffat interprets the data with a rapidly rotating 0 star in a $\mathrm{WN} 6+09$ system. The $\mathrm{O}$-star absorption lines are superposed on the spectrum of the WR star.

A major argument for spherical winds has come from the interpretation of the flat-topped profiles (section 3). However, pure rotation plus high turbulence also produces flat-topped profiles. Underhill \& Nemec (1989) calculated profiles from a rotating, hollow ring with a turbulent velocity of 280 $\mathrm{km} / \mathrm{s}$ which illustrates this point. High turbulent velocities have recently also been introduced to explain the edge velocities of P-Cygni absoption troughs of UV resonance lines (e.g., Prinja, Barlow \& Howarth 1990).

If WR stars are surrounded by Be-star-like rotating disks, one might expect to see double-peaked profiles. While some evidence for double-peaked line profiles has been furnished by, e.g., Underhill et al. (1990) and Vreux et al. (1992), it is not very abundant. This might be due to the high outflow-torotational speed ratio in WR winds. Theoretical work on line profiles originating in non-spherical WR winds has been scarce. Rumpl (1980) provided fits to line profiles of WR6 with an expanding (but not rotating), equatorial wind using a two-component velocity law. For certain viewing angles, excellent results could be obtained. The line profiles resulting from a rotating and expanding wind have been calculated by Mazalli (1990), and are qualitatively indistinguishable from those calculated using spherical outflows. I conclude that fits to the emission-lines profiles are as yet not a unique measure of the wind geometry. The true profile shapes in single WR stars must depend on the relative contributions of outflow, rotational, and turbulent speeds at different distances in WR winds. (Note that the profiles in candidate WR+c systems such as WR6 can also be explained via interactions of the WR wind with its companion, $c f$. Koenigsberger, these proceedings).

There are five WR stars with ring nebulae which may contain stellar ejecta (Chu 1991): S308 around the WN5 star WR6, RCW104 around the WN6 star WR75, NGC6888 around the WN6 star WR136, M1-67 which surrounds the WN8 star WR124, and RCW58 surrounding the WN8 star WR40. Their shapes might provide circumstantial evidence for wind asym- 
metries. The nebula around WR6 is nearly circular, but has a hernia at $330^{\circ}$, which looks like a blow-out. The WR75 nebula is highly filamentary. The nebulae around WR136, WR40, and WR124 are cleary axisymmetric. Nota et al. (these proceedings) obtained coronographic images and spectra of the inner M1-67 nebula around WR124. They find that the nebula is clumpy and bipolar. The axial symmetry is also present in the velocity distribution of the blobs.

A few individual WR stars have been proposed to have flattened winds. WR6 and WR134 were suggested to have flattened winds based on spectropolarimetry (Schulte-Ladbeck et al. 1990, 1991, 1992). WR134 has independently been proposed to have an axisymmetric wind from spectroscopy by Underhill et al. (1990) and Vreux et al. (1992). Underhill \& Fahey (1987b) claim a disk in WR139; my spectropolarimetric observations of this object do not support a disk in WR139 at this time, but more data are being collected. Becker \& White (these proceedings) presented eight years of radio observations of the WC7+O binary WR140. To interpret the light-curve they suggest that the wind of the WR star is in a disk.

\section{Spectropolarimetry}

The light from any distant star is polarized due to interstellar polarization (ISP). To discriminate intrinsic, linear polarization against ISP, one can search for temporal variability (ISP is constant) or the line effect: changes of polarization accross the emission lines in the spectrum of a WR star. An example for the line effect in the spectrum of the WN8 star WR40 is given in Figure 1. If the line emission is intrinsically unpolarized, it determines the ISP of the WR star at one wavelength. The vector difference between the line and its underlying continuum polarization, the line-to-continuum vector, yields the position angle of the electron distribution thus providing a diagnostic of the geometry. The line polarization can also be used to define the origin of the coordinate system in which the temporal variations occur (which may have been monitored in several broad-band filters), and is a similar geometric probe. During my talk, I showed a large number of $\mathrm{Q}-\mathrm{U}$ diagrams to illustrate the temporal behavior of the line-to-continuum vector in many stars. These are not included here because of the limited space. We are currently working on a summary paper which will present all of these results (Schulte-Ladbeck, Hillier \& Nordsieck, in preparation).

Let me note that, while it is fairly easy to detect deviations from spherical geometry with polarimetry, for example, one detection of a line-effect is sufficient, it is more challenging to determine the geometry of the scattering material. To do so, several observations of a given star are required. The signal-to-noise ratio needed to find the line effect is high (3000 for a polarization error of $0.3 \%$ per resolution element), and it is difficult to obtain 


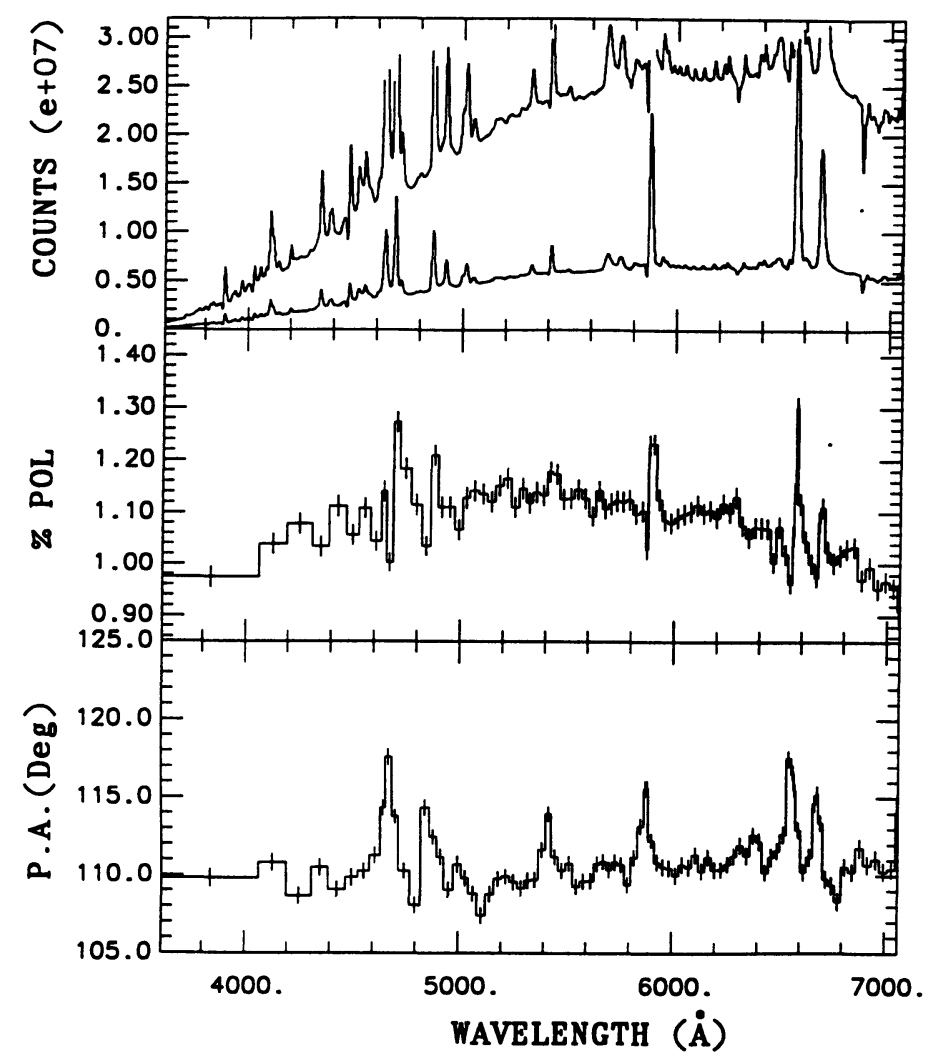

Fig. 1. The observed spectrum of the counts, percentage polarization and position angle of WR40. The errors of the polarization are based on photon counting statistics, and are $\pm 1 \sigma$ in size.

enough observing time at a large telescope for these temporal studies.

The principal wind geometries tested with spectropolarimetry can be classed into four categories. First, the winds could be spherically symmetric and homogeneous, as is preferred by theorists. This case seems to be realized in nature, in the WC5 star WR111. There is no line effect and no polarization variability (St-Louis et al. 1987) in this star. Therefore, polarization observations support that there are spherical and stable winds.

Secondly, the wind could have a time-averaged, spherical structure but could be locally inhomogeneous. This is the case in the WN8 star WR40. The polarization scatters in the $\mathrm{Q}-\mathrm{U}$ plane about the line datum which determines the ISP; hence there is no preferred symmetry axis in the wind.

Thirdly, a wind could be aspherical but homogeneous. This geometry could be noticed if a WR star showed the line effect, but no temporal polarization variations. We have no detection of such a case. 
Fourthly, the winds of WR stars could be aspherical and inhomogeneous. Examples for this geometry are WR6 and WR134 (see Schulte-Ladbeck et al. 1990, 1991, 1992). The Luminous Blue Variable AG Car, which was recently classed as a WN11 star by Smith, Crowther \& Prinja (1994) also displays the spectropolarimetric characterisitics of a time- and latitude-dependent outflow (Schulte-Ladbeck et al. 1994).

The polarization across emission line profiles may furnish an additional, new tool to investigate the wind structure. An example WR profile for WR6 was given by Schulte-Ladbeck, Meade \& Hillier (1992). Radiative-transfer model profiles for Be stars have been published by Pockert \& Marlborough (1978), and support a disk geometry. Hillier (these proceedings) is currently developing a code which uses his "Standard Model" as input and then predicts the polarization across line profiles of WR stars.

\section{Summary}

There is evidence from spectropolarimetry of two types of wind asymmetries in WR stars. First, the winds of many stars are inhomogeneous, permeated with electron-density enhancements which we have come to term blobs or clumps. Second, there is evidence for rotation in a few WR stars from the geometry of their winds.

Evidence for rotationally flattened winds has now been searched for in 34 of the catalogued WR stars (cf. Schulte-Ladbeck 1994, Table 1), but only two can be interpreted as having latitude-dependent winds. A third example is AG Car, an LBV/WN11 star. Spectropolarimetric results suggest that WR stars may not all be rapid rotators, although a spectrum of rotational speeds may exist.

Since most WR stars surveyed do not show a line effect, spectropolarimetric observations do not provide support for the Luminous Magnetic Rotator model as the solution to the "momentum problem".

O-type stars rotate, and some evolutionary descendants, the LBVs and the $\mathrm{B}[\mathrm{e}]$ stars, show evidence for latitude-dependent winds (e.g., SchulteLadbeck et al. 1994). This indicates that significant angular momentum is being lost when stars enter the WR phase. Among the flat-wind candidates, AG Car is a WNL star and may be considered a young WR star. WR6 and WR134 are WNE stars, thus somewhat evolved WR stars. Therefore, some stars, possibly the ones with the most rapidly rotating main-sequence progenitors, are able to retain their angular momentum into advanced WR stages. Note also that there is no spectropolarimetric detection of a flattened wind in a WC star, suggesting that any remaining angular momentum is lost in the WN stage. An evolutionary track with rotation was presented by Fliegner \& Langer (these proceedings), indicating that a comparison between theory and observation might soon be possible. 


\section{Acknowledgements}

This work was supported by NASA grant NAWG-2977. I am grateful to the AAS for a travel grant.

\section{References}

Annuk, K. 1991, in: K.A. van der Hucht \& B. Hidayat (eds.), Wolf-Rayet Stars and Interrelations with other Massive Stars in Galaxies, Proc. IAU Symp. No. 143 (Dordrecht: Kluwer), p. 245

Aslanov, A.A., Cherepashchuk, A.M. 1990, Sov. Astron. 34, 602

Bjorkman, J.E., Cassinlli, J.P. 1993, ApJ 409, 429

Castor, J.I. 1979, in: P.S. Conti \& C.W.H. de Loore (eds.), Mass Loss and Evolution of O-type Stars, Proc. IAU Symp. No. 83 (Dordrecht: Reidel), p. 175

Castor, J.I., Abbott, D.C., Klein, R.I. 1975, $A p J$ 195, 157

Chu, Y.-H. 1991, in: K.A. van der Hucht \& B. Hidayat (eds.), Wolf-Rayet Stars and Interrelations with other Massive Stars in Galaxies, Proc. IAU Symp. No. 143 (Dordrecht: Kluwer), p. 349

Crowther, P.A., Hillier, D.J., Smith, L.J. 1994 AEA submitted

Kuhi, L.V. 1973, in: M.K.V. Bappu \& J. Sahade (eds.), Wolf-Rayet and High-Temperature Stars, Proc. IAU Symp. No. 49 (Dordrecht: Reidel), p. 205

Lucy, L.B., Abbott, D.C. 1993, ApJ 405, 738

Lucy, L.B., Solomon, P.M. 1970, ApJ 159, 879

Massey, P. 1980, ApJ 236, 526

Mazzali, P.A. 1990, $A \cup A$ 238, 191

Owocki, S.P., Cranmer, S.R., Blondin, J.M. 1994, ApJ 424, 887

Poe, C.H., Friend, D.B., Cassinelli, J.P. 1989, ApJ 337, 888

Prinja, R.K., Barlow, M.J., Howarth, I.D. 1990, $A p J$ 361, 607

Poeckert, R., Marlborough, J.M. 1978, ApJ 220, 940

Robert, C. 1991, PhD thesis, Université de Montréal

Rumpl, W.M. 1980, ApJ 241, 1055

Schmid-Burgk, J. 1982, $A \& A$ A 108, 169

Schmutz, W. 1990, in: L.A. Willson \& R. Stalio, Angular Momentum and Mass Loss for Hot Stars, Nato ASI Series C 316 (Dordrecht: Kluwer), p.227

Schulte-Ladbeck, R.E., Nordsieck, K.H., Nook, M.A., Magalhães, A.M., Taylor, M., Bjorkman, K.S., Anderson, C.M. 1990, ApJ (Letters) 365, L19

Schulte-Ladbeck, R.E., Nordsieck, K.H., Taylor, M., Nook, M.A., Bjorkman, K.S., Magalhães, A.M., Anderson, C.M. 1991, ApJ 382, 301

Schulte-Ladbeck, R.E., Nordsieck, K.H., Taylor, M., Bjorkman, K.S., Magalhães, A.M., Wolff, M.J. 1992, ApJ 387, 347

Schulte-Ladbeck, R.E., Meade, M.R., Hillier, D.J. 1992, in: L. Drissen, C. Leitherer \& A. Nota (eds.), ASP Conf. Ser. 22, 118

Schulte-Ladbeck, R.E. 1994, in: N. St.-Louis, A.W. Fullerton, A.F.J. Moffat et al. (eds.), Instability and Variability of Hot-Star Winds, in press

Schulte-Ladbeck, R.E., Clayton, G.C., Hillier, D.J., Harries, T.J., Howarth, I.D. 1994, ApJ in press

Smith, L.J., Crowther, P.A., Prinja, R.K. 1994, $A \& A$ 281, 833

St-Louis, N., Drissen, L., Moffat, A.F.J., Bastien, P. 1987, ApJ 322, 870

Underhill, A.B., Fahey, R.P. 1987a, $A p J$ 300, 379

Underhill, A.B., Fahey, R.P. 1987b, $A p J 313,358$

Underhill, A.B., Nemec, A.F.L. 1989, $A p J$ 345, 1008

Underhill, A.B., Gilroy, K.K., Hill G.M., Dinshaw, N. 1990, ApJ 351, 666

Vreux, J.-M. Gosset, E., Bohannan, B., Conti, P.S. 1992, A\&A 256, 148 


\section{DISCUSSION:}

Cherepashchuk: Your new polarimetric results on V444 Cyg are extremely interesting. Could you describe in some more detail the shape of WR extended atmosphere which follows from your observational data:

Schulte-Ladbeck: First, the difference in polarization between the continuum and the HeII $\lambda$ 4686 line is evidence that the continuum and the line originate from different regions. According to Hillier, if one considers the theoretical W-R ionization structure alone (i.e. pretend that there is no $O$ star, no colliding winds) then one expects the HeII $\lambda 4686$ emission to come from a region which lies outside of the orbit).

Second, the Brown, McLean \& Enslie binary polarization model assumes point sources illuminating a distribution of electrons. Here, first harmonic variations in polarization are caused by material outside of the orbital plane and distributed asymmetrically about it (c.f. their figures at $\mathrm{i} \approx 85^{\circ}$, with certain $\mathrm{A}=\mathrm{H} / \mathrm{G}$ ). However, the HeII light source is extended in V444 Cyg and it is puzzling that the amplitude of the continuum variations, and that the sense of the Q-U loop is opposite to that of the continuum. We should both work on models of binary polarization with extended light sources (c.f. Rodrigues \& Magalhaes, this volume) and obtain more spectropolarimetry of V444 Cyg. Observationally, this system presents a difficult problem because we really need a big telescope (to get enough photons) for a large number of nights (to obtain the phase coverage). We will certainly continue to monitor it at Pine Bluff Observatory, but progress will be slow.

Owocki: Just a point of clarification. I missed what the timescale is for those observed polarization variations, and whether there is any evidence for periodicity.

Schulte-Ladbeck: The timescales are not well sampled observationally. The spectropolarimetric data have been gathered at the Pine Bluff Observatory and the AngloAustralian Telescope over several years. There are 'broad-band' polarimetry data available (cf. the papers by Drissen, Robert, or St-Louis) which have been obtained during single, extended $(\sim 1$ month) runs and where certain targets were revisited every night. Periodicities were only noticed in known WR + $\mathrm{O}$ binaries.

Pollock: How disappointed were you to see nothing from WR140? If the colliding-wind models people have been calculating are right, there should be gross asymmetries in the distribution of mass. Is it therefore surprising nothing was observed?

Schulte-Ladbeck: Yes, I was VERY disappointed. After all, we monitored this star for 8 years! Yes, we expected to see asymmetries in the distribution of the gas and the dust. However, there was no change of the optical, lines polarization with time, no difference between the polarization in HeII $\lambda 4686$ and the underlying continuum polarization, and the combined polarization spectrum from 1989-1994 can be modelled extremely well with interstellar polarization alone; the residuals are consistent with zero intrinsic polarization after the interstellar polarization is subtracted from the observed polarization (c.f. Schulte-Ladbeck et al., this volume).

Possible explanations for these non-detections include: a) the material was distributed spherically symmetrically (highly unlikely considering the wind collision at periastron passage); b) the polarization from the dust cloud created at 1993 periastron passage was small and was swamped by the direct light from the W-R plus $\mathrm{O}$ star; c) the continuum and the HeII $\lambda 4686$ line scattered in the same way.

I'm looking forward to the paper by Becker \& White on Friday. Their technique obviously had greater sensitivity to wind asymmetries in the WR 140 system. 
Moffat: You mentioned that WR $138=\mathrm{HD} 193077$ (WN6 + abs) has broad absorption lines implying rapid rotation of the WR star. However, this star appears to be a WR + $\mathrm{O}$ binary of long period, $\mathrm{P} \sim 5$ yrs (Lamontagne et al., Annuk - Bali Symposium), in which the absorption lines arise in a normal (but rapidly rotating) late-type O-star. I have also looked at the WR + abs system globally (Moffat et al. 1986, AJ). The result is that most WNE + abs systems are in fact WR + O binaries, while most WNL + abs stars have (often slightly blue-shifted) absorption lines intrinsic to the WR star.

Williams: When observing WR 140, did you find any change in the raw line polarization during the orbit?

Schulte-Ladbeck: No. The line polarization (HeIl $\lambda$ 4686) did not vary in time since 1989, i.e., since we had an instrument capable of measuring it. There is also no variation with wavelength. The difference between the polarization of the line and the continuum is zero (within the observational errors). Cf. also Schulte-Ladbeck et al., this volume.

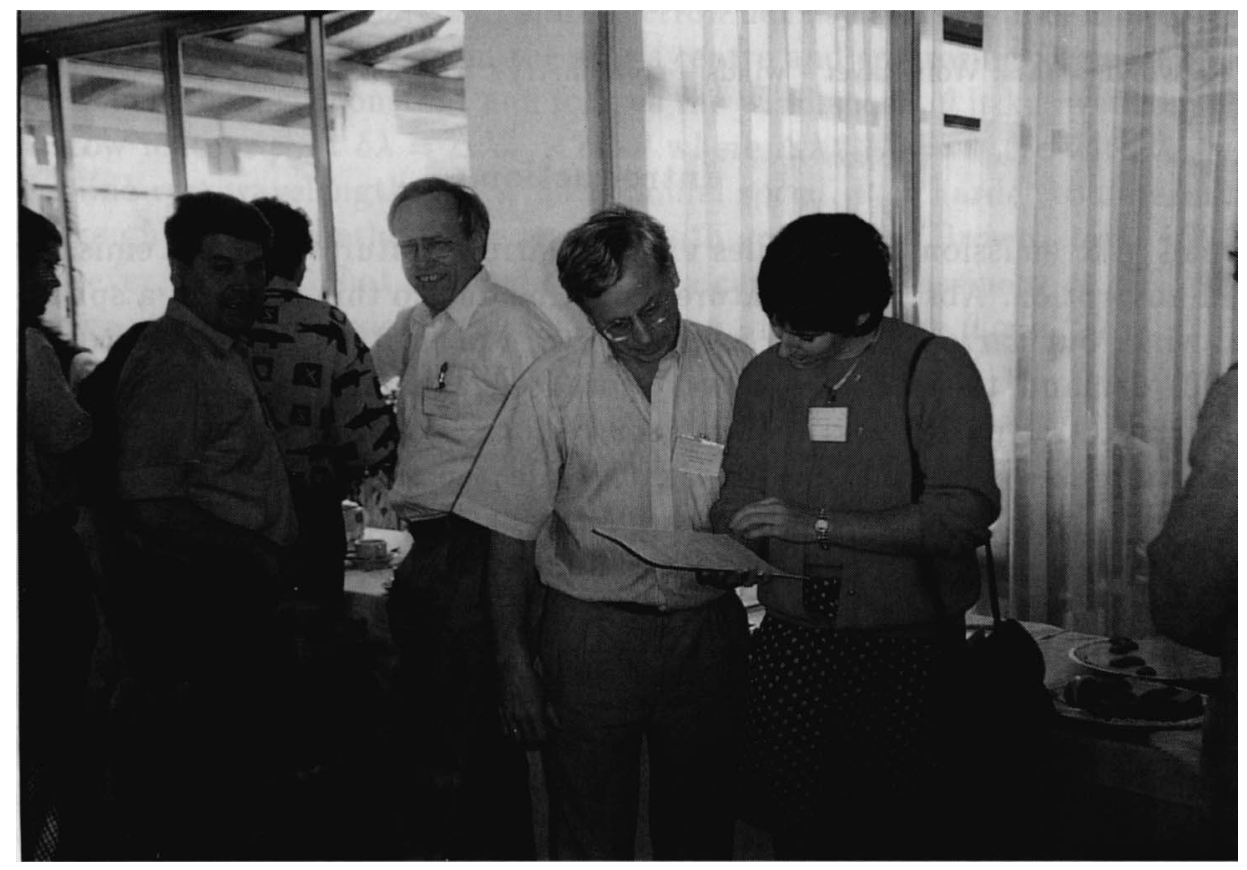

Cherepashchuk, Cassinelli, van der Hucht, Schulte-Ladbeck 